\title{
A nonlinear viscoelastic bushing element in multibody dynamics
}

\author{
R. Ledesma, Z.-D. Ma, G. Hulbert, A. Wineman
}

\begin{abstract}
This paper presents a formulation for incorporating nonlinear viscoelastic bushing elements into multibody systems. The formulation is based on the assumption that the relaxation function can be expressed as a sum of functions which are nonlinear in deformation and exponentially decreasing in time. These forces can represent elastomeric mounts or bushings in automotive suspension systems. The numerical implementation of the nonlinear viscoelastic bushing model into a general purpose rigid multibody dynamics code is described, and an extension of the formulation is also presented wherein component flexibility is included. Model validation was performed by comparing experimental data to simulation results obtained using the nonlinear viscoelastic model and a nonlinear elastic model. The experimental data were obtained at the Center's facilities by testing an automotive lower control arm/bushing system, subjected to a simulated road load event. The comparison demonstrates the better load prediction capability of the viscoelastic bushing model compared to the conventional model.
\end{abstract}

\section{1}

\section{Introduction}

The use of multibody dynamics analysis (MDA) as a viable tool in computer-aided engineering has received wide acceptance in many engineering and manufacturing industries, especially in the automative industry. The recognition of the potential of MDA as a CAE tool is due to tremendous strides in the development of this field of study as evidenced by the large number of publications on the subject during the last decade. Recent developments in this field cover a wide range of issues including the choice of coordinates which describe the dynamic system (Unda, Garcia, Losantos, Esperantza 1987), the choice of methods for the formulation of the equations of motion

\section{Communicated by S. N. Atluri, 9 October 1995}

R. Ledesma, Z.-D. Ma, G. Hulbert, A. Wineman Center for Automotive Structural Durability Simulation, Department of Mechanical Engineering and Applied Mechanics, The University of Michigan, 2250 G.G. Brown, Ann Arbor, MI 48109-2125, USA

\section{Correspondence to: Z.-D. Ma}

The authors gratefully acknowledge the support of this research by Ford Motor Company to the Center for Structural Durability Simulation at the University of Michigan. The authors thank John Holmes, Shixiang Shi, Samir Kakli and Jing Ling for building the bushing and RPC test facilities and for conducting the experiments and to Tim Van Dyke for assistance with the bushing data reduction procedures.
(Bayo and Avello 1994), the development of algorithms for the efficient numerical solution of the equations of motion (Bayo and Ledesma 1994; Park and Chiou 1993), the modeling of material properties and mechanical constraints (Xie and Amirouche 1994; Ambrosio and Nikravesh 1992), the proper modeling of component flexibility (Wallrapp and Schwertassek 1991; Banerjee 1993; Ryu and Kim 1994; Boutaghou, Erdman, Stolarski 1992), among others. The unifying objective of these developments is to enhance the ability of multibody dynamics simulation to predict the actual behavior of the dynamic system that is being modeled. The ultimate goal is to have a general-purpose multibody dynamics code that can predict the behavior of a dynamic system during the design stage so that the performance of the system can be evaluated before expensive prototypes are built.

This paper describes developing a proper model of bushing forces between suspension system components. The study was motivated by engineers at Ford Motor Company whose experience have led them to conclude that modeling bushing forces in automotive suspension systems plays an important role in predicting the dynamic behavior of the suspension system. The choice of the bushing model is especially crucial in predicting loads that act on the suspension system components which are supported by bushings. Accurate prediction of the dynamic loads acting on the suspension system components is important because these loads feed directly into the fatigue life prediction of the components. Considering the modeling of bushing forces in the multibody dynamics codes, the present state-of-the-art is to model the bushing forces as a Kelvin solid which is represented by a spring in parallel with a viscous damper. The spring force is a nonlinear function of the instantaneous deformation of the bushing and the viscous damping force is a linear function of the instantaneous relative velocity of the two components that are connected by the bushing. One main drawback of this approach is that the nonlinear elastic bushing model provides a dynamic response in which the energy dissipation is a linear function of the excitation frequency. Hence, the nonlinear elastic bushing model will damp out the high frequency content of the dynamic response and will, in general, capture the correct energy dissipation characteristic of the material at only one particular excitation frequency. Experimental results indicate that bushings, which are made of elastomeric material, exhibit a deformation history-dependent behavior that can be characterized as one with 'fading memory'. These results suggest that the bushing response fits nicely into the theory of nonlinear viscoelasticity. The history-dependent, nonlinear viscoelastic force model for the bushing force is characterized by a convolution integral where the kernel is a series of exponential 
functions which can capture the 'fading memory' characteristic of the polymeric material in the bushing.

Previous studies on the dynamic analysis of structures containing viscoelastic material have been reported in the literature (Holzlohner 1974; Yamada, Takabatake, Sato 1974; Johnoson and Kienholz 1982; Morman and Nagtegaal 1983; Bagley and Torvik 1985; Golla and Hughes 1985; Xie, Roystre, Ciskowski 1989; Gaul and Chen 1993; Yi and Hilton 1994). Most of these studies deal with the viscoelastic behavior at the material level and use finite elements or boundary elements to build up the viscoelastic structure and subsequently determine the dynamic response either through the frequency domain or Laplace domain, or through a time domain realization of the equations of motion in the Laplace domain. Because of the use of the finite element method or the boundary element method to model the viscoelastic structure, all of the aforementioned studies require expensive calculations and are not suitable for the dynamics of multibody systems which are made up of several rigid, elastic, or viscoelastic components. A notable exception is the work by Gaul and Chen (1993) who used the boundary element method to develop a relation between 12 stress resultants and 12 deformation variables which represent an elastomeric mount in a multibody system. That study, however, was limited to linear viscoelastic behavior so that the correspondence principle could be used to replace the elastic isotropic material behavior with the corresponding viscoelastic constitutive equations. In contrast, the present study efficiently captures the nonlinear viscoelastic behavior by characterizing the behavior of the elastomeric bushing not at the material level, but rather, at the component level where stress resultants are expressed as nonlinear functions of the deformation variables. This approach bypasses expensive finite element or boundary element calculations, so that the method can be readily applied to multibody system dynamics simulation (at the expense, however, of some predictive capabilities).

The paper is structured as follows. We describe the formulation of the bushing forces as nonlinear viscoelastic force elements in multibody systems and the numerical implementation of the formulation into the general-purpose multibody dynamics code ADAMS (Mechanical Dynamics, Inc.). The verification of the formulation and code implementation was performed by building a simple multibody system at the Center's test facility and comparing simulation results with experimental data. The simple multibody system consists of an automotive suspension system component which is supported by bushings and subjected to prescribed dynamic loads and boundary conditions. The proposed nonlinear viscoelastic bushing model is also compared with the conventional nonlinear elastic bushing model. Comparison between the two bushing models shows that the nonlinear viscoelastic bushing model more accurately correlates with the experimental results.

\section{2}

\section{Formulation of the equations of motion}

The proposed bushing model takes the form of a set of uncoupled nonlinear viscoelastic force elements. In the context of multibody dynamics, these nonlinear viscoelastic forces are treated as massless nonlinear force elements that act between two parts or bodies which are connected by a bushing. The formulation and implementation of these massless nonlinear viscoelastic force elements can be best explained by first looking at the equations of motion for a constrained set of rigid bodies, and subsequently determining how the bushing forces enter into the equations of motion.

Consider a simple rigid multibody system consisting of two bodies, $i$ and $j$, which are subject to mechanical constraints. The equations of motion for the rigid multibody system may be written in the following descriptor form:

$$
\left[\begin{array}{ll}
\mathbf{m}_{R R} & \mathbf{m}_{R \theta} \\
\mathbf{m}_{\theta R} & \mathbf{m}_{\theta \theta}
\end{array}\right]\left\{\begin{array}{l}
\ddot{\mathbf{R}} \\
\ddot{\boldsymbol{\theta}}
\end{array}\right\}+\left[\begin{array}{l}
\boldsymbol{\phi}_{R}^{t} \\
\boldsymbol{\phi}_{\theta}^{t}
\end{array}\right]\{\boldsymbol{\lambda}\}=\left\{\begin{array}{l}
\mathbf{Q}_{R} \\
\mathbf{Q}_{\theta}
\end{array}\right\}+\left\{\begin{array}{l}
\mathbf{F}_{R} \\
\mathbf{F}_{\theta}
\end{array}\right\}
$$

subject to the holonomic constraint equations

$\phi(\mathbf{R}, \boldsymbol{\theta})=0$

In Eqs. (1) and (2), the generalized coordinates ( $R, \theta)$ refer to the rigid body translation and rigid body orientation, respectively. The rigid body coordinates describe the position and orientation of the body-fixed frames attached to each part or body in the multibody system, as shown in Fig. 1. Also in Eq. (1), $\mathbf{m}_{R R}$ and $\mathbf{m}_{\theta \theta}$ are the mass matrices associated with the translational mass and rotational inertia of the rigid body, respectively, and $\mathbf{m}_{R \theta}$ is the inertial coupling between the rigid body translation and rigid body rotation. The force vector $Q$ is the generalized force due to external forces, and the force vector $F$ is the generalized force due to quadratic velocity terms including centrifugal forces. The matrix $\left[\phi_{R}, \phi_{\theta}\right]$ is the constraint Jacobian matrix, and the vector $\lambda$ is the vector of Lagrange multipliers associated with the constraints. The equations of motion, Eqs. (1) and (2), form a set of differential algebraic equations (DAE's) which are, in general, numerically more difficult to solve than ordinary differential equations (ODE's).

Now consider the two bodies $i$ and $j$ to be connected to each other by a bushing whose attachment points are located at point $P^{i}$ on body $i$ and point $P^{j}$ on body $j$ as shown in Fig. 1 . If the bushing forces are treated as massless force elements acting between bodies $i$ and $j$, the only way that these force elements can enter the equations of motion is through the generalized force vector $\mathbf{Q}$ by treating the bushing forces as external forces applied to the bodies which are connected by a bushing. In what

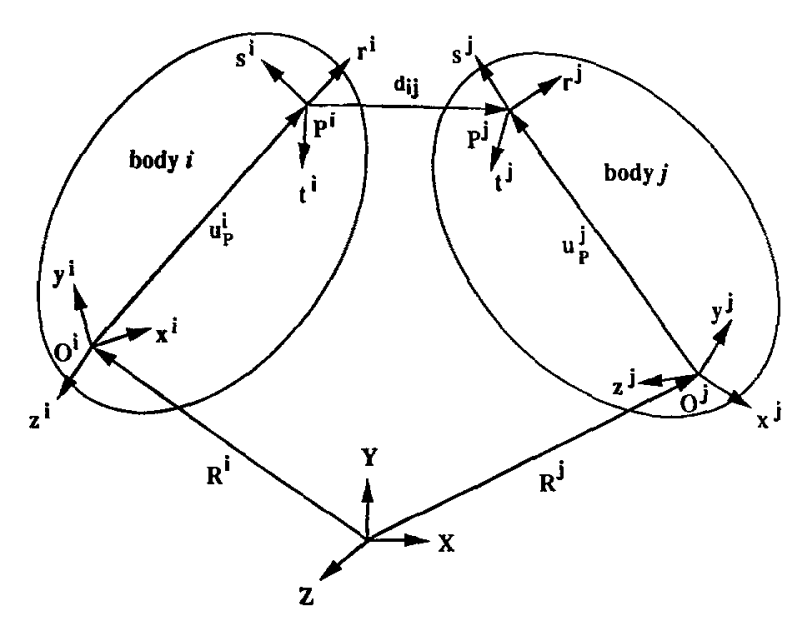

Fig. 1. Multibody system and reference frames 
follows, we will consider only bushing forces in the radial and axial directions to simplify the discussion. A similar procedure will apply for the conical and torsional bushing moments. If we consider a bushing force vector $f_{i}$ acting on body $i$ and whose components are measured with respect to the bushing coordinate system $r^{2}-s^{2}-t^{2}$, the generalized force vector associated with the translational coordinates of body $i$ due to the bushing force vector $f_{\imath}$ is given by

$\mathbf{Q}_{R}^{l}=\mathbf{A}_{\imath} \mathbf{A}_{p} \mathbf{f}_{s}$

and the generalized force vector associated with the rotational coordinates of body $i$ due to the bushing force vector $\mathrm{f}_{2}$ is given by

$\mathbf{Q}_{\theta}^{t}=\mathbf{G}_{t}^{t} \tilde{\mathbf{u}}_{P}^{i} \mathbf{A}_{P} \mathbf{f}_{t}$

where $A_{p}$ is the orthogonal transformation matrix from the bushing coordinate system to the body-fixed reference frame, $A_{t}$ is the orthogonal transformation matrix from the body-fixed reference frame to the inertial reference frame, and $\boldsymbol{u}_{p}^{l}$ is the position of point $P^{i}$ measured with respect to the body-fixed reference frame. The tilde symbol above the vector $\mathbf{u}_{p}^{t}$ represents the skew-symmetric matrix operator, and $G_{i}$ is a matrix that maps the time derivatives of the orientation coordinates to the angular velocity of the body, and is defined by the equation

$\boldsymbol{\omega}_{l}=\mathbf{G}_{\imath} \dot{\boldsymbol{\theta}}_{t}$

where $\omega_{i}$ is the angular velocity of body $i$ and whose components are measured relative to the body-fixed reference frame. In Eq. (3), the generalized force vector associated with the rigid body translational coordinates is simply the transformation of the bushing force from the bushing coordinate system to the inertial reference frame, while in Eq. (4), the generalized force vector associated with the rigid body orientation coordinates characterizes the moment of the bushing force vector about the origin of the body-fixed reference frame.

Considering the bushing force vector $\mathrm{f}_{i}$, the bushing force is modeled as a set of nonlinear viscoelastic forces which depend not only on the instantaneous bushing deformation, but also on the history of the bushing deformation. The nonlinear viscoelastic bushing is based on a modified Pipkin-Rogers superposition principle (Pipkin and Rogers 1968) where the nonlinear viscoelastic force is given by

$\mathbf{f}_{t}(t)=\hat{\mathbf{R}}\left[\boldsymbol{\Lambda}_{t}(t), 0\right]+\int_{0}^{t} \frac{\partial \hat{\mathbf{R}}\left[\boldsymbol{\Delta}_{t}(s), t-s\right]}{\partial(t-s)} d s$

in which $\mathrm{f}_{t}$ is the bushing force vector measured with respect to the bushing coordinate system, $\Delta_{i}$ is the bushing deformation vector measured with respect to the bushing coordinate system, and $\hat{\mathbf{R}}$ is the relaxation function which characterizes the bushing's viscoelastic response. The first term on the R.H.S. of Eq. (6) represents the instantaneous response of the bushing, while the second term on the R.H.S. of Eq. (6) represents the history-dependent response of the bushing force. The bushing deformation vector $\boldsymbol{A}_{i}$, whose components are measured with respect to the bushing coordinate system, is related to the global bushing deformation vector by the following transformation:

$\Delta_{i}=\mathbf{A}_{p}^{t} \mathbf{A}_{i}^{t} \mathbf{d}_{i j}$

where $\mathbf{d}_{i j}$ is the bushing deformation measured with respect to the inertial reference frame, and the orthogonal matrices $A_{P}$ and $A_{i}$ are the bushing coordinate system-to-body reference frame and body reference frame-to-inertial reference frame transformation matrices, respectively. The global bushing deformation vector $\mathbf{d}_{i j}$ is in turn related to the generalized coordinates through the following relation:

$\mathbf{d}_{\imath\}}=\left\{\left(\mathbf{R}^{\jmath}+\mathbf{A}_{j} \mathbf{u}_{P}^{\prime}\right)-\left(\mathbf{R}^{i}+\mathbf{A}_{i} \mathbf{u}_{P}^{i}\right)\right\}$

where $\mathbf{R}^{i}$ and $\mathbf{R}^{j}$ are the position vectors of the origins of the body-fixed reference frames attached to bodies $i$ and $j$, respectively, $A_{3}$ and $A_{j}$ are the body reference frame-to-inertial reference frame transformation matrices for bodies $i$ and $j$, respectively, and $\mathbf{u}_{p}^{i}$ and $\mathbf{u}_{p}^{f}$ are the position vectors of the bushing attachment points on bodies $i$ and $j$, measured with respect to their respective body-fixed reference frames. Equations (8), (7), (6), (4), and (3) form the algorithmic sequence for computing the generalized forces due to bushing forces acting on body $i$. A similar procedure applies for computing the generalized forces due to the bushing forces acting on body $j$.

\section{3}

\section{Uniaxial nonlinear viscoelasticity}

Thus far, we have made no assumptions on the mathematical description of the nonlinear viscoelastic bushing force, except that the bushing force can be decomposed into an instantaneous force response and a history-dependent force response characterized by a convolution integral, the kernel of which is the time derivative of the vector of relaxation functions as depicted in Eq. (6). As a first attempt, we can assume that the bushing force components are decoupled, i.e., radial forces, axial forces, torsional moments, conical moments, and their work-conjugate deformation components are independent. With this assumption, the viscoelastic bushing force can be decomposed into two force components along two orthogonal radial directions and a force component along the axial direction. Similarly, the bushing moments can be decomposed into two conical moments about the two orthogonal radial directions and a torsional moment about the axial direction. Furthermore, the bushing force or moment components depend only on their associated work-conjugate deformation component. Using the above assumption, each component of the nonlinear viscoelastic

force-displacement relation of Eq. (6) simplifies to the uniaxial nonlinear viscoelastic force-displacement relation, which can be written as the following scalar equation:

$f(t)=R[\Delta(t), 0]+\int_{0}^{t} \frac{\partial R[\Delta(s), t-s]}{\partial(t-s)} d s$ 
where $f(t)$ is the viscoelastic bushing force component along a radial or axial direction, and $\Delta(t)$ is the corresponding bushing deformation along the same direction. The scalar function $R[\Delta(s), t-s]$ is the relaxation function of the bushing force component along the given direction. We further assume that the relaxation function can be expressed as the sum of integer powers of the bushing deformation where each term in the series is multiplied by a time-dependent function,

$R[\Delta(s), t-s]=\sum_{k=1}^{N} \Delta^{k}(s) G_{k}(t-s)$

where each of the time-dependent functions $G_{k}(t-s)$ is expressed as a series of exponential terms,

$G_{k}(t-s)=g_{k 0}+\sum_{j=1}^{N_{k}} g_{k} \exp \left[-(t-s) / \tau_{k j}\right]$

Combining Eqs. (9) -(11), we obtain the following expression for the nonlinear viscoelastic bushing force along a particular direction:

$F(t)=\sum_{k=1}^{N} \Delta^{k}(t) G_{k}(0)-\sum_{k=1}^{N} \sum_{j=1}^{N_{k}} \frac{g_{k j}}{\tau_{k j}} \int_{0}^{t} \exp \left[-(t-s) / \tau_{k j}\right] \Delta^{k}(s) d s$

where each of the stiffness coefficients in the instantaneous force response is given by

$G_{k}(0)=\sum_{j=0}^{N_{k}} g_{k j}$

The parameters $N, N_{k}, g_{k 0}, g_{k j}$, and $\tau_{k j}\left(j=1, N_{k}\right.$ and $\left.k=1, N\right)$ are bushing material parameters that are determined from experiments and processed through an extrapolation procedure. The bushing force component of Eq. (12) is calculated by applying the trapezoidal rule to compute the convolution integral, resulting in the following discrete time approximation of the bushing force:

$F\left(t_{n+1}\right)=\sum_{k=1}^{N} \Delta^{k}\left(t_{n+1}\right) G_{k}(0)-\sum_{k=1}^{N} \sum_{j=1}^{N_{k}} \frac{g_{k j}}{\tau_{k j}} \tilde{I}_{k j}^{n+1}$

where $\widetilde{I}_{k j}^{n+1}$ is the numerical approximation to the convolution integral and computed from the following recursive equation:

$$
\begin{aligned}
\tilde{I}_{k j}^{n+1}= & \widetilde{I}_{k j}^{n} \exp \left(-\left(t_{n+1}-t_{n}\right) / \tau_{k j}\right) \\
& +\left[\Delta^{k}\left(t_{n}\right) \exp \left(-\left(t_{n+1}-t_{n}\right) / \tau_{k \jmath}\right)+\Delta^{k}\left(t_{n+1}\right)\right]\left(\frac{t_{n+1}-t_{n}}{2}\right)
\end{aligned}
$$

with the recursion started by setting

$\widetilde{I}_{k j}^{0}=0$

The recursive algorithm for the numerical computation of the nonlinear viscoelastic force obviates the need to store the entire deformation history of the bushing, thus improving the computational efficiency in the calculation of the bushing forces. It is worthwhile to note that the recursive algorithm is possible only because we have chosen to use exponential terms in the time-dependent functions $G_{k}(t-s)$.

The computation of the viscoelastic bushing forces is carried out in ADAMS through a user-written subroutine.

4

\section{Validation}

To validate the implementation of the nonlinear viscoelastic bushing force element into the multibody dynamics code, the bushing model was employed in ADAMS using a user-written subroutine. We then modeled a simple multibody system consisting only of two bodies, namely, an automotive suspension system component and the ground, wherein the two bodies are connected by bushings. Two bushing models are used for comparison: the conventional nonlinear elastic model and the proposed nonlinear viscoelastic bushing model, hereafter referred to as NLE and VISCO, respectively. The material parameters for both models were obtained from a suite of tests performed for every bushing. For this purpose, a special bushing testing machine was built at the Center. The bushing testing machine has two linear actuators which can provide axial and radial forces along the bushing's principal directions, and two rotary actuators which can provide torsional and conical bushing moments. The bushing testing machine was used in conducting static tests, harmonic tests, ramp tests, and simulated road load tests on each bushing to determine its time-dependent response. A parameter identification procedure was employed to process the test results and to derive a set of bushing material parameters for direct use in Eq. (12).

To validate the results of the simulation, an experimental set-up of the automotive suspension system component was built at the Center, including a remote parameter control (RPC) system. The experiment consists of a lower control arm supported by two bushings and subjected to prescribed dynamic loads and displacements at the ball joint attachment to the spindle. Load cells were attached to the bushings to measure the forces transmitted across the bushings. In addition, LVDT's were attached at the outboard end of each bushing to measure bushing deformation. Dynamic tests were conducted and the experimental results were compared with the simulation results obtained from the VISCO and NLE bushing models.

A finite element model of the lower control arm is shown in Fig. 2, wherein the bushing supports at the front and rear ends are illustrated. The ball joint at the lateral end is subjected to prescribed dynamic loads along the fore-aft and lateral directions and a prescribed displacement in the vertical direction. The prescribed forces and displacements can be seen as insets in Fig. 2. The duration of the event is 1.8 seconds. The maximum peak-to-peak applied force along the fore-aft direction is $13.4 \mathrm{kN}$ and the maximum peak-to-peak applied force along the lateral direction is $13.2 \mathrm{kN}$. The maximum peak-to-peak prescribed displacement along the vertical direction is $46.7 \mathrm{~mm}$. These three time-varying inputs were used in all the tests and simulations.

Figures 3 and 4 show the fore-aft bushing forces for the front and rear bushings, respectively, obtained from the RPC test, VISCO simulation, and NLE simulation. The maximum difference, in terms of peak-to-peak forces, between the 

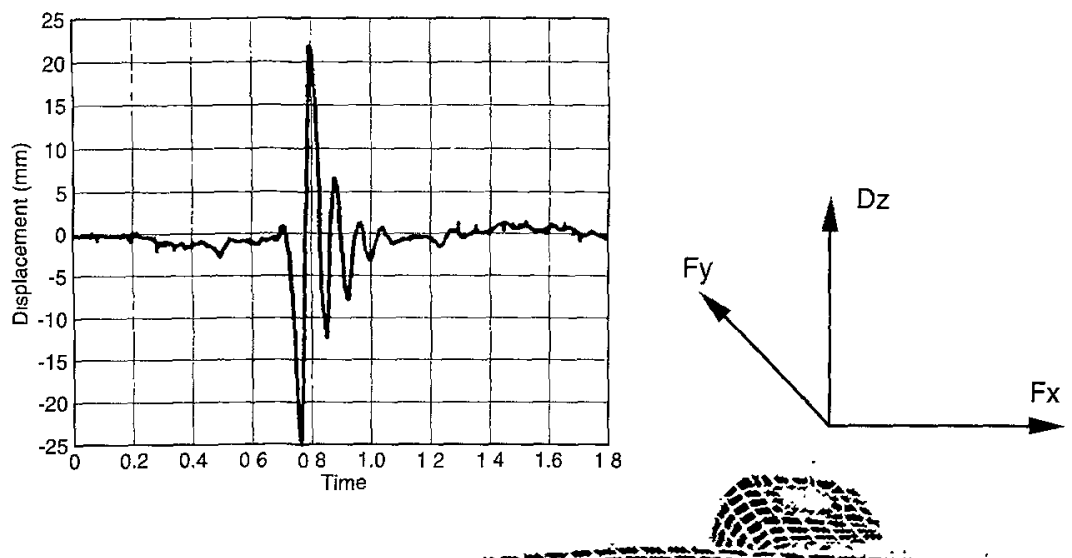

ireforto

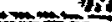
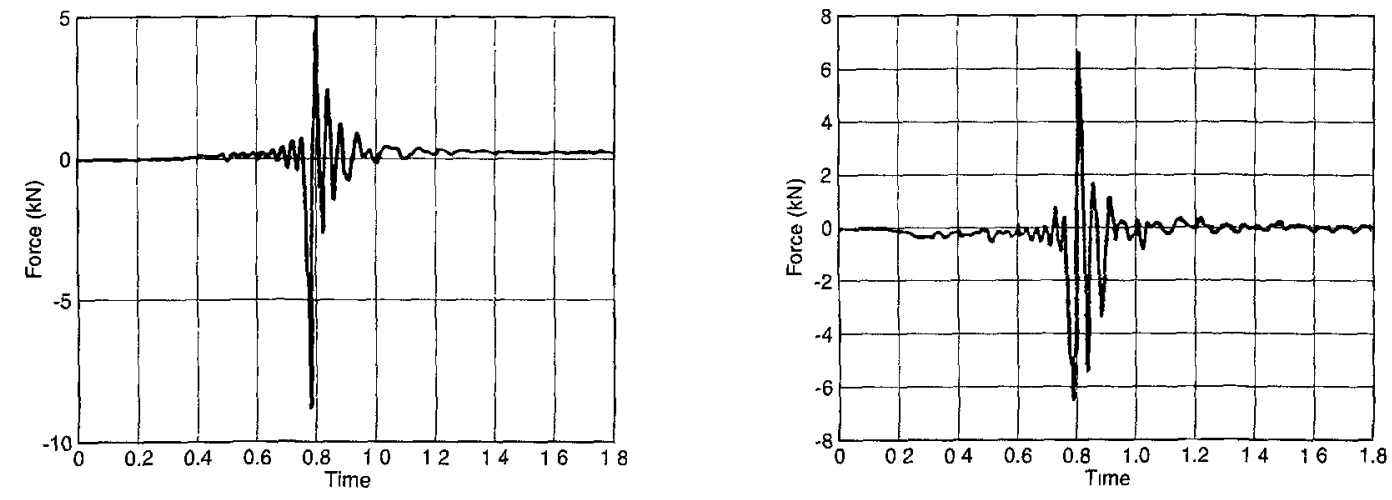

(

Fig. 2. Lower control arm and prescribed forces annd displacements at the ball joint

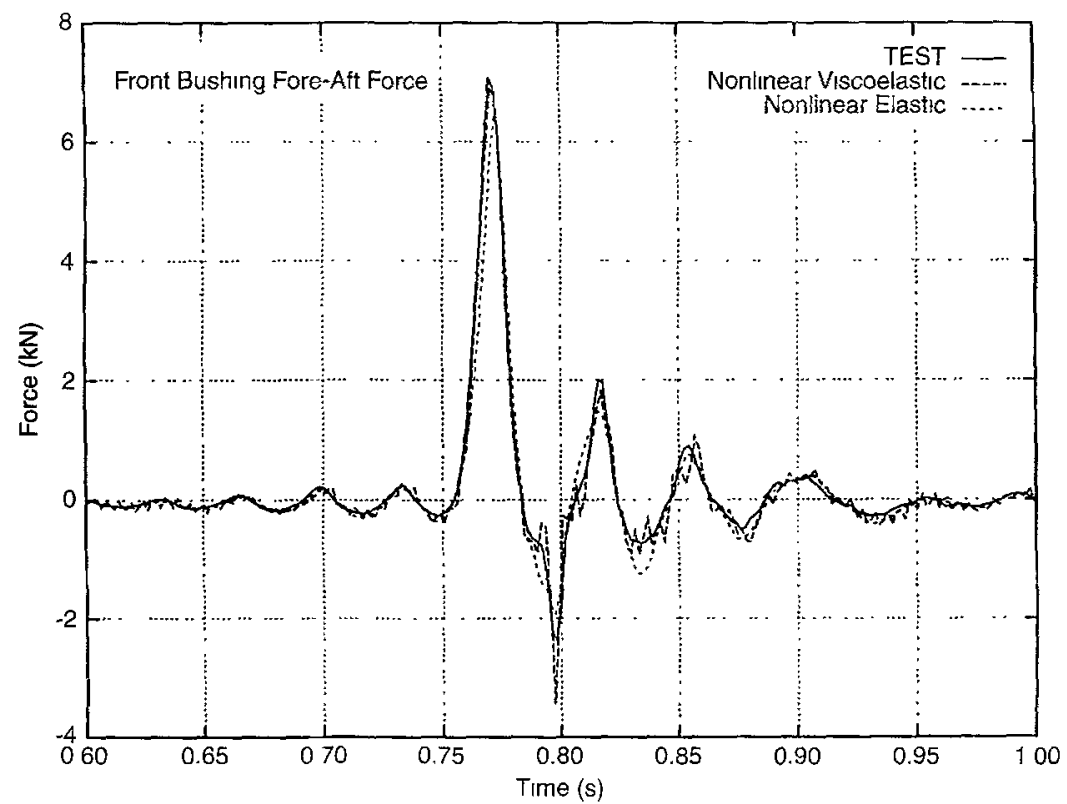

Fig. 3. Front bushing fore-aft force: comparison between test results, nonlinear viscoelastic bushing model, and nonlinear elastic bushing model nonlinear viscoelastic bushing model and the nonlinear elastic bushing model is approximately $15 \%$, while the maximum difference between the nonlinear viscoelastic bushing model and the measured test data is approximately $12 \%$. Using the NLE model, the high-frequency response is damped out because the linear viscous damper provides energy dissipation that linear increases with frequency. High-frequency response is not observed in the RPC test data because of limitations in the 


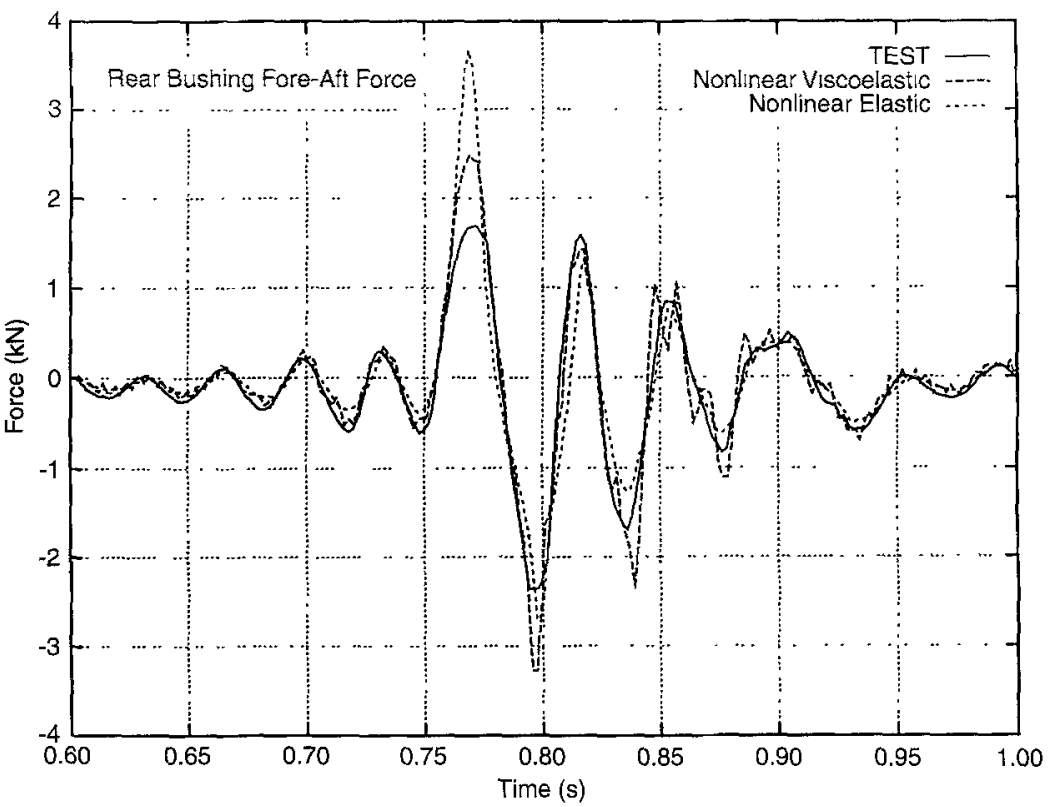

Fig. 4. Rear bushing fore-aft force: comparison between test results, nonlinear viscoelastic bushing model, and nonlinear elastic bushing model
Table 1. Comparison between simulation and test results: maximum peak-to-peak bushing forces

\begin{tabular}{|c|c|c|c|}
\hline Force Component & ADAMS-NLE & ADAMS-VISCO & Test Results \\
\hline $\begin{array}{l}\text { Front Bushing } \\
\text { Fore-Aft Direction }\end{array}$ & $8.396 \mathrm{kN}$ & $10.349 \mathrm{kN}$ & $9.503 \mathrm{kN}$ \\
\hline $\begin{array}{l}\text { Front Bushing } \\
\text { Lateral Direction }\end{array}$ & $10.807 \mathrm{kN}$ & $12.399 \mathrm{kN}$ & $10.969 \mathrm{kN}$ \\
\hline $\begin{array}{l}\text { Rear Bushing } \\
\text { Fore-Aft Direction }\end{array}$ & $6.384 \mathrm{kN}$ & $5.753 \mathrm{kN}$ & $4.056 \mathrm{kN}$ \\
\hline $\begin{array}{l}\text { Rear Bushing } \\
\text { Lateral Direction }\end{array}$ & $12.077 \mathrm{kN}$ & $12.408 \mathrm{kN}$ & $12.600 \mathrm{kN}$ \\
\hline
\end{tabular}

sampling rate of the load cells. Table 1 summarizes the comparison of bushing forces obtained from NLE simulation, VISCO simulation, and test results. From this table we observe that the predicted nonlinear viscoelastic bushing forces obtained from the VISCO model are closer to the test results than those obtained from the NLE model.

Figure 5 shows the rear bushing displacement along the fore-aft direction obtained from the RPC test, VISCO simulation, and NLE simulation. As expected, the nonlinear viscoelastic bushing model shows higher peaks in the predicted bushing displacement compared to the nonlinear elastic bushing model. Again this is attributed to the fact that the conventional nonlinear elastic model damps out the high frequency response. The VISCO model captures the frequency of the measured bushing displacement whereas the NLE model shows errors in amplitude and phase with respect to the measured displacements. Table 2 summarizes the comparison of the maximum peak-to-peak displacement response obtained from NLE simulation, VISCO simulation, and test results. It can be seen that the nonlinear viscoelastic bushing model gives a better estimate of the measured bushing displacement than the nonlinear elastic bushing model. However, the predicted bushing displacements obtained from the multibody system dynamics simulation do not agree well with the measured displacements; this discrepancy can be as high as
$90 \%$. The discrepancy is due to the fact that component flexibility has been ignored in the formulation of the equations of motion. This contention is supported by calculations performed using the fully nonlinear finite element code ABAQUS (Karlssen and Sorensen, Inc.) wherein the flexibility of the control arm is considered. A comparison of dynamic responses between a rigid body model and a flexible body model of the lower control arm is shown in the time history plots of Figs. 6 and 7. The finite element calculations show that there is load redistribution due to the flexibility of the component, so that the front bushing experiences larger peak-to-peak displacements and the rear bushing experiences smaller peak-to-peak displacements, consistent with the experimental results. The results of the finite element analysis also indicate that the predicted bushing forces can be improved if component flexibility is taken into account. Figures 8 and 9 clearly show the load redistribution due to component flexibility. These figures also show the high frequency response due to the vibration of the flexible component. The finite element calculations, which required a fully nonlinear dynamic analysis due to the relatively large vertical motion imposed at the ball joint, required computation times which are orders of magnitude higher compared to that of the multibody system dynamic analyses.

\section{5}

\section{Extension to flexible multibody system dynamics}

Based on the validation results in the previous section, the assumption of rigid bodies in multibody dynamics is adequate if bushing forces are the only concern in the dynamic simulation. However, if the displacements of some points of interest are required from the dynamic simulation, the assumption of rigid bodies is no longer adequate even for relatively stiff components such as the lower control arm. In this regard, we present an extension of the formulation of bushing forces to the dynamics of flexible multibody systems. Similar to the formulation of the generalized forces due to bushing forces in the equations of motion for rigid multibody systems, we first present the equations of motion for a flexible 


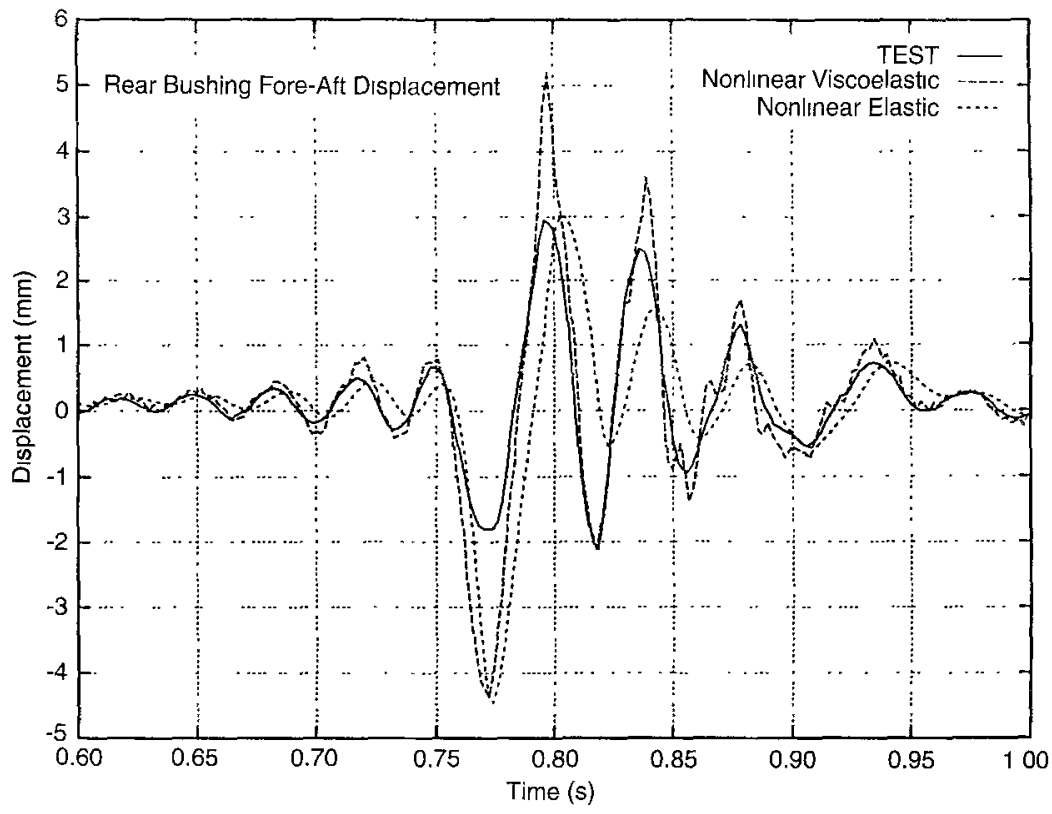

Table 2. Comparison between simulation and test results: maximum peak-to-peak displacements

\begin{tabular}{|c|c|c|c|}
\hline $\begin{array}{l}\text { Displacement } \\
\text { Component }\end{array}$ & ADAMS-NLE & ADAMS-VISCO & Test Results \\
\hline $\begin{array}{l}\text { Front Bushing Disp. } \\
\text { Fore-Aft Direction }\end{array}$ & $6.796 \mathrm{~mm}$ & $8.705 \mathrm{~mm}$ & $11.762 \mathrm{~mm}$ \\
\hline $\begin{array}{l}\text { Front Bushing Disp. } \\
\text { Lateral Direction }\end{array}$ & $8.104 \mathrm{~mm}$ & $10.439 \mathrm{~mm}$ & $10.716 \mathrm{~mm}$ \\
\hline $\begin{array}{l}\text { Rear Bushing Disp. } \\
\text { Fore-Aft Direction }\end{array}$ & $7.460 \mathrm{~mm}$ & $9.522 \mathrm{~mm}$ & $5.041 \mathrm{~mm}$ \\
\hline $\begin{array}{l}\text { Rear Bushing Disp. } \\
\text { Lateral Direction }\end{array}$ & $0.790 \mathrm{~mm}$ & $1.296 \mathrm{~mm}$ & $1.861 \mathrm{~mm}$ \\
\hline
\end{tabular}

multibody system, and subsequently introduce the generalized forces due to the bushing forces that act on a deformable body in the multibody system.

Consider now the bodies $i$ and $j$ in Fig. 1 to be deformable bodies. The equations of motion for the flexible multibody system can be written in the following descriptor form (Shabana 1989):

Rear bushing fore-aft displacement comparison between test results, nonlinear iscoelastic bushing model, and nonlinear elastic bushing model

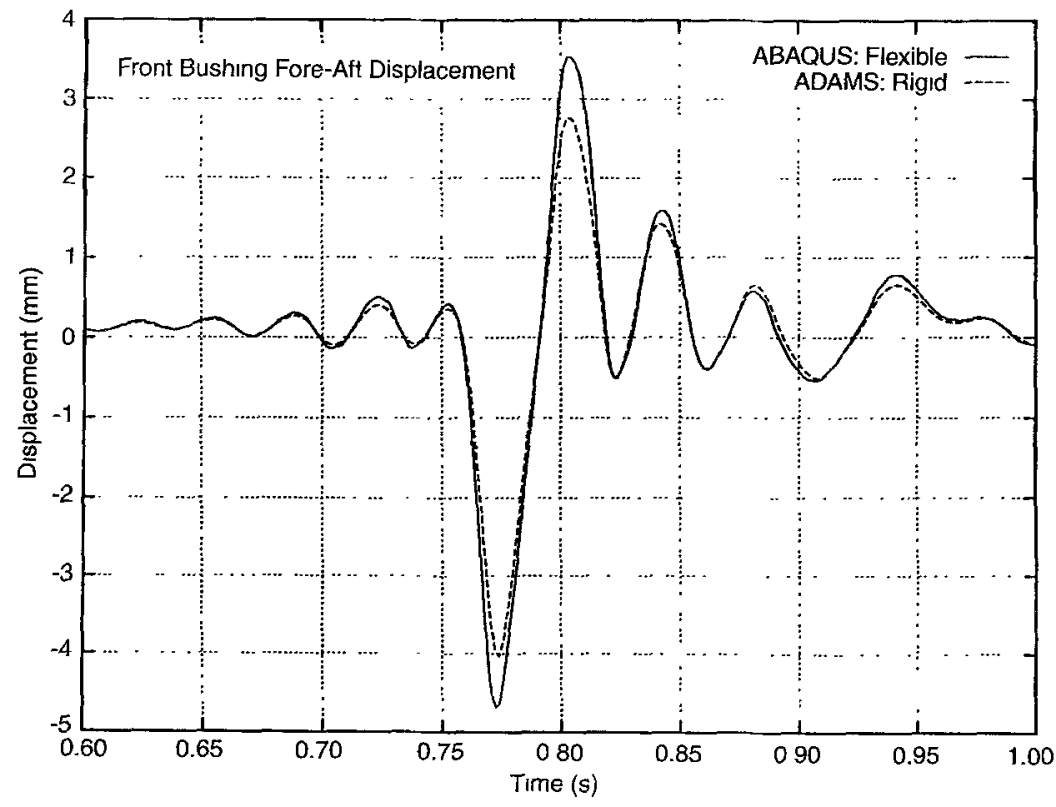

Fig. 6. Front bushing fore-aft displacement: comparison between flexible lower control arm and rigid lower control arm 

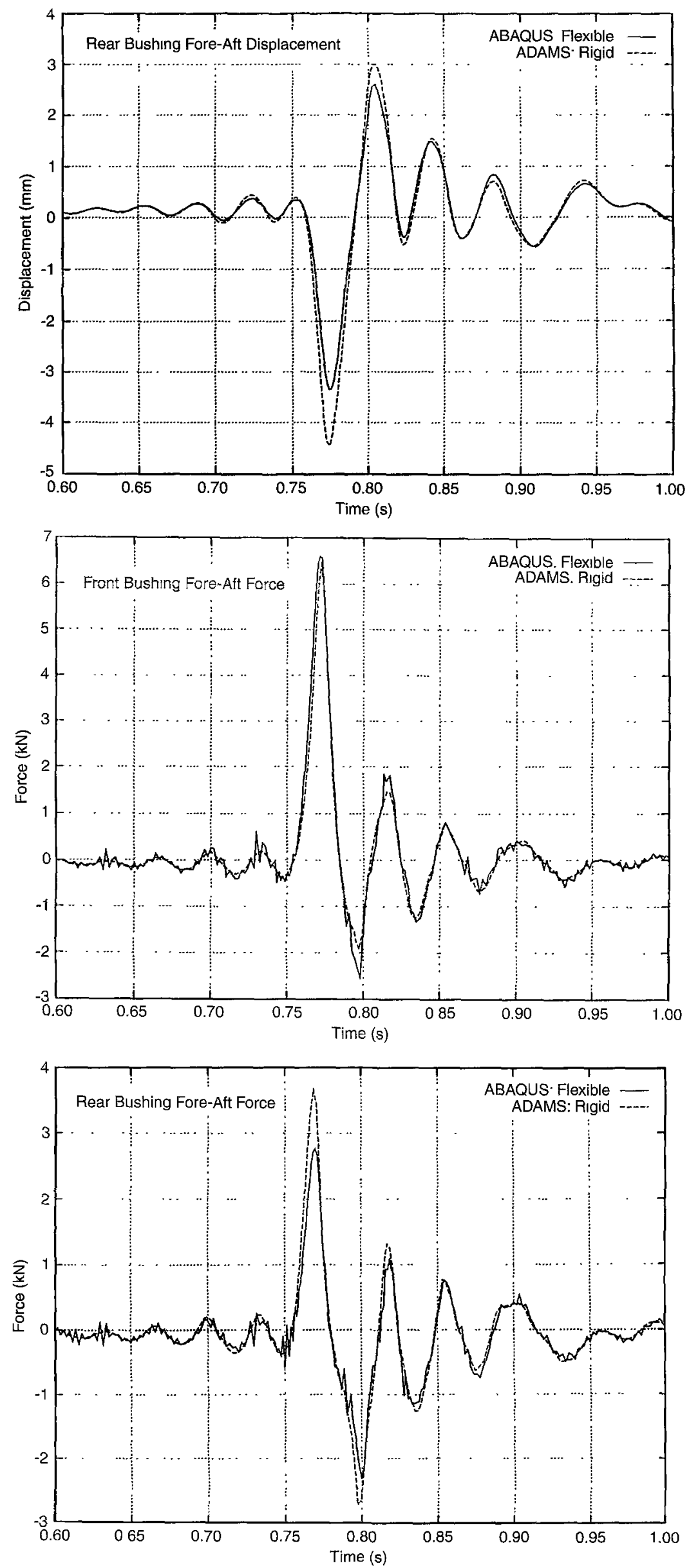

Fig. 7. Rear bushing fore-aft displacement: comparison between flexible lower control arm and rigid lower control arm

Fig. 8. Front bushing fore-aft force: comparison between flexible lower control arm and rigid lower control arm

Fig. 9. Rear bushing fore-aft force: comparison between flexible lower control arm and rigid lower control arm 
subject to the holonomic constraint equations

$\phi\left(\mathbf{R}, \boldsymbol{\theta}, \mathbf{q}_{f}\right)=0$

where the generalized coordinates $\left(\mathbf{R}, \boldsymbol{\theta}, \mathbf{q}_{f}\right)$ refer to the translation of the body reference frame, the orientation of the body reference frame, and the deformation of the flexible body, respectively. The mass matrix is a function of the orientation of the body reference frame and the elastic deformation. The force vector $Q$ refers to the generalized forces due to externally applied loads, and the force vector $F$ includes the centrifugal forces as well as the Coriolis forces arising from the elastic deformation. The equations of motion as expressed in Eq. (17) are based on the assumption that small elastic deformations are superposed on the overall rigid body motion. The force vector $\mathbf{P}_{f}$ refers to the elastic forces within the deformable body. This force vector can be decomposed into a force vector due to the (linear) structural stiffness matrix and a force vector due to a stress-dependent stiffness matrix which captures the stiffening effects from the rigid body rotation.

Similar to the case of rigid multibody systems, the bushing forces enter the equations of motion through the generalized force vector $Q$ by treating the bushing forces as external forces that act between two bodies or parts that are connected by a bushing. The generalized force vector due to the bushing forces will be of the same form as that of the generalized bushing force vectors formulated for the case of rigid multibody systems, with some minor modifications that account for the elastic deformation of the bodies which are connected by the bushing. Again, in the following discussion, we will consider only the contribution of radial and axial bushing forces to the generalized bushing force vector. The contribution of conical and torsional bushing moments to the generalized bushing force vector can be easily derived by applying the principle of virtual work.

If we consider a bushing force vector $\mathrm{f}_{i}$ acting on deformable body $i$ and whose components are measured with respect to the bushing coordinate system $r^{i}-s^{i}-t^{i}$ at the bushing attachment point $P^{2}$, the generalized bushing force vector associated with the translation of the body reference frame of body $i$ is given by

$\mathbf{Q}_{R}^{t}=\mathbf{A}_{t}\left[\mathbf{I}+\tilde{\mathbf{\Omega}}_{p}\right] \mathbf{A}_{P} \mathbf{f}_{t}$

where $\boldsymbol{A}_{p}$ is the rotation transformation matrix from the bushing coordnate system to the body reference frame in the undeformed configuration, $\boldsymbol{\Omega}_{P}$ is the skew-symmetric matrix whose associated axial vector is the elastic rotation vector at the bushing attachment point. Again, Eq. (19) is valid only for small elastic rotations. The generalized bushing force vector associated with the rotation of the body reference frame of body $i$ is given by

$\mathbf{Q}_{\theta}^{i}=\mathbf{G}_{i}^{t} \tilde{\mathbf{u}}_{p}^{2}\left[\mathbf{I}+\widetilde{\mathbf{\Omega}}_{p}\right] \mathbf{A}_{P} \mathbf{f}_{t}$

where $\mathbf{u}_{p}^{2}$ is the instantaneous position vector of the bushing attachment point measured with respect to the body reference frame. Unlike the case of rigid bodies, $\mathbf{u}_{p}^{i}$ is not constant since it now depends on the elastic deformation of the deformable body. When a finite element discretization or an assumed mode formulation is used for the spatial interpolation of the flexible body's deformation, the position vector of the bushing attachment point can be expressed as

$\mathbf{u}_{p}^{i}=\left(\mathbf{u}_{p}^{i}\right)_{r}+\mathbf{N}_{p} \mathbf{q}_{f}^{i}$

where the first term $\left(\mathbf{u}_{p}^{t}\right)_{r}$ is the position vector of the bushing attachment point in the undeformed configuration and the second term is the elastic deformation of the bushing attachment point, defined in terms of the interpolation or shape function matrix $\mathbf{N}_{P}$ evaluated at point $P^{\prime}$, and the vector of generalized elastic coordinates $\mathbf{q}_{f}^{2}$. Finally, the generalized bushing force vector associated with the deformation coordinates of body $i$ is given by

$\mathbf{Q}_{f}^{i}=\mathbf{N}_{p}^{t}\left[\mathbf{I}+\tilde{\mathbf{\Omega}}_{p}\right] \mathbf{A}_{p} \mathbf{f}_{i}$

The nonlinear viscoelastic bushing force vector $\mathrm{f}_{t}$ is expressed as a function of the bushing deformation history

$\mathbf{f}_{l}(t)=\hat{\mathbf{R}}\left[\boldsymbol{\Delta}_{t}(t), 0\right]+\int_{0}^{t} \frac{\partial \hat{\mathbf{R}}\left[\boldsymbol{\Delta}_{l}(s), t-s\right]}{\partial(t-s)} d s$

where the local bushing deformation vector is related to the global bushing deformation vector through the transformation relation

$\mathbf{\Delta}_{i}=\mathbf{A}_{p}^{t}\left[\mathbf{I}+\tilde{\mathbf{\Omega}}_{p}\right]^{t} \mathbf{A}_{t}^{t} \mathbf{d}_{t}$

and the global bushing deformation vector is determined from the generalized coordinates through the following equation:

$\mathbf{d}_{i j}=\left\{\left(\mathbf{R}^{j}+\mathbf{A}_{j}\left[\left(\mathbf{u}_{p}^{3}\right)_{r}+\mathbf{N}_{p}^{j} \mathbf{q}_{f}^{3}\right]\right)-\left(\mathbf{R}^{i}+\mathbf{A}_{1}\left[\left(\mathbf{u}_{F}^{2}\right)_{r}+\mathbf{N}_{p}^{2} \mathbf{q}_{f}^{i}\right]\right)\right\}$

Equations (25), (24), (23), (19), (20) and (22) form the algorithmic sequence for the computation of the generalized force vectors due to bushing forces acting on body $i$. A similar procedure is needed to determine the generalized force vectors due to bushing forces acting on body $j$.

\section{6}

\section{Conclusion}

In this paper, we have presented the formulation of nonlinear viscoelastic bushing forces as massless force elements between two bodies in a multibody system. The numerical implementation of the proposed formulation into the general-purpose multibody dynamics code ADAMS was completed for rigid multibody systems. Validation of the resulting model was performed by comparing results obtained from a nonlinear viscoelastic bushing model and a nonlinear elastic bushing model to those obtained from measured test results. The code validation and comparison with measured data revealed that the nonlinear viscoelastic bushing model gives a more accurate prediction of dynamic loads and displacements than the nonlinear elastic bushing model. However, the rigid multibody system models did not accurately predict the displacements of specific points of interest. The 
cause of the poor displacement prediction capabilities of the rigid multibody dynamics models was traced to the fact that component flexibility plays an important role in the displacement response, and also to a lesser degree, in the prediction of dynamic loads. To achieve better prediction capabilities, the formulation of nonlinear viscoelastic bushing forces has been extended to include deformable bodies connected by bushings in the context of general flexible multibody systems. The proposed formulation of nonlinear viscoelastic force elements in flexible multibody systems can be easily implemented in multibody dynamics codes that include the capability for modeling flexible components.

\section{References}

ABAQUS: User's Manual, Habbitt, Karlsson and Sorensen, Inc., Pawtucket, Rhode Island

Shabana, A. 1989: Dynamics of Multibody Systems. Wiley

ADAMS: (Automatic Dynamic Analysis of Mechanical Systems)

Reference Manual, Mechanical Dynamics, Inc., Ann Arbor, Michigan

Ambrosio, J.; Nikravesh, P. E. 1992: Elasto-plastic deformations in multibody dynamics. Nonlinear Dynamics. 3: 85-104

Bagley, R.; Torvik, P. 1985: Fractional calculus in the transient analysis of viscoelastically damped structures. AIAA Journal, 23: 918-925

Banerjee, A. K. 1993: Block-diagonal equations for multibody elastodynamics with geometric stiffness and constraints. Journal of Guidance, Control, and Dynamics. 16: $1092-1100$

Bayo, E.; Avello, A. 1994: Singularity-free augmented Lagrangian algorithms for constrained multibody dynamics. Nonlinear Dynamics. 5: 209-231

Bayo, E.; Ledesma, R. 1994: Augmented Lagrangian and projection methods for constrained multibody dynamics. Proceedings of the 23rd ASME Biennial Mechanisms Conference, Design Engineering Division. DE-72: 237-247

Boutaghou, Z.; Erdman, A.; Stolarski, H. 1992: Dynamics of flexible beams and plates in large overall motions. ASME Journal of Applied Mechnics. 59: 991-999

Gaul, L.; Chen, C. 1993: Modeling of viscoelastic elastomer mounts in multibody systems. In: Schiehlen, W. (ed). Advanced Multibody System Dynamics, pp. 257-276. Kluwer Academic Publishers
Golla, D.; Hughes, P. 1985: Dynamics of viscoelastic structures - a time domain finite element formulation. ASME Journal of Applied Mechanics. 52: 897-906

Holzlohner, U. 1974: A finite element analysis for time-dependent problems. International Journal for Numerical Methods in Engineering. 8: 55-69

Johnson, C.; Kienholz, D. 1982: Finite element prediction of damping in structures with constrained viscoelastically damped structures. AIAA Journal. 20: 1284-1290

Morman, K.; Nagtegaal, J. 1983: Finite element analysis of sinusoidal small-amplitude vibrations in deformed viscoelastic solids, part 1: theoretical development. International Journal for Numerical Methods in Engineering. 19: 1079-1103

Park, K.; Chiou, J. 1993: Discrete momentum-conserving explicit algorithm for rigid body dynamic analysis. International Journal for Numerical Methods in Engineering. 36: 1071 - 1083

Pipkin, A.; Rogers, T. 1968: A non-linear integral representation for viscoelastic behavior. Journal of Mechanics and Physics of Solids. 16: 59-72

Ryu, J.; Kim, S. 1994: General approach to stress stiffening effects on flexible multibody dynamic systems. Mechanics of Structures and Machines. 22: 157-180

Yamada, Y.; Takabatake, H.; Sato, T. 1974: Effect of time-dependent material properties on dynamic response. International Journal for Numerical Methods in Engineering. 8: 403-414

Unda, J.; Garcia de Jalon, J.; Losantos, F.; Esperantza, R. 1987:

A comparative study on some different formulations of the dynamic equations of constrained mechanical systems. ASME Journal of Mechanisms, Transmissions and Automation in Design. 109: 466-474

Xie, M.; Amirouche, F. M. L. 1994: Treatment of creep and nonlinearities in flexible multibody dynamics. AIAA Journal. 32: 190-197

Wallrapp, 0.; Schwertassek, R. 1991: Representation of geometric stiffening in multibody system simulation. International Journal for Numerical Methods in Engineering. 32: 1833-1850

Xie, K.; Roystre, L.; Ciskowski, R. 1989: A boundary element method formulation for fractional operator modeled viscoelastodynamic structures. In: Advances in Boundary Elements, Vol. 3, Stress Analysis, pp. 55-64. Computational Mechanics Publications

Yi, S.; Hilton, H. 1994: Dynamic finite element analysis of viscoelastic composite plates in the time domain. International Journal for Numerical Methods in Engineering. 37: 4081-4096 